\title{
Simulation of transient blood flows in the artery with an asymmetric stenosis
}

\author{
B. Wiwatanapataphee ${ }^{1} \quad$ Y.-H. $\mathrm{Wu}^{2}$ \\ S. Amornsamankul ${ }^{3} \quad$ B. Novaprateep ${ }^{4}$
}

(Received 29 August 2006; revised 1 April 2008)

\begin{abstract}
This article focuses on the transient behaviour of blood flow in stenotic arteries. Human blood is modelled as an incompressible nonNewtonian fluid. A numerical technique based on the finite element method is developed to simulate the blood flow taking into account of the transient periodic behaviour of the blood flow in cardiac cycles. The flow pattern, the distribution of pressure and the wall shear stresses, are computed. The results show that the pulsatile pressure and the time variation of wall shear rate have patterns similar to that for the pulsatile velocity during the cardiac cycles. On the back toe of the stenosis there exists a small recirculation region which causes the direction of the wall shear stress in some part to oscillate, likely leading to atherosclerotic disease. The back toe is thus an ideal location for applying a clot dissolving drug.
\end{abstract}

See http://anziamj.austms.org.au/ojs/index.php/ANZIAMJ/article/view/107 for this article, (c) Austral. Mathematical Soc. 2008. Published May 6, 2008. ISSN 14468735 


\section{Contents}

1 Introduction

C1007

2 Mathematical model

C1008

3 Method of solution

C1011

4 Numerical results and discussion

C1012

5 Conclusions

C1018

References

C1019

\section{Introduction}

The blood circulatory system consists of parts such as the heart, the arterial and the venous systems as well as the microcirculatory systems. The presence of unusual hemodynamic condition in the arteries often creates abnormal biological responses. Skewing of the blood speed in some region could cause oscillating direction of wall shear stress which can create pockets leading to atherosclerotic disease. The skewing of velocity tends to be localized and results in the narrowing of the artery lumen - a stenosis [1]. In the arteries with high grade stenoses, very high shear stresses near the throat of the stenosis can activate the platelets. This induces thrombosis, and may totally block the blood flow to the heart and lead to heart attacks and strokes.

In order to understand the genesis of coronary diseases, over the past two decades, a number of mathematical models have been proposed to describe the rheological behavior of blood in stenotic arteries. Most models assume that blood acts as a Newtonian fluid with constant viscosity and that the vessel is rigid. Models of this type do not provide a satisfactory description of 
the dynamics of real pulsatile blood flow in the artery. Mann and Tarbell [2] used a non-Newtonian model to determine a nonlinear dependence of the viscosity of blood on the strain rate. Their model was intended to study the flow of fluid analog of blood in rigid curved and straight arteries. Grigioni et al. [3] investigated the wall shear stress and velocity field in an in vivo experiment of unsteady vascular dynamics. They used a non-Newtonian model to describe an unsteady flow in a rigid pipe driven by a known oscillatory pressure gradient.

Although blood flow has been modelled by many researchers, there have only been a few numerical studies on the flow in stenotic arteries using the realistic pulsatile flow conditions on the inlet and outlet [4]. In this article a mathematical model is developed to study the unsteady state blood flow through a stenotic artery of different severity. Blood is modelled as a nonNewtonian fluid. Using the straight tube (as shown in Figure 1) having three different size of stenosis, $50 \%, 65 \%$ and $75 \%$, numerical simulations are carried out for the flow field, temperature field, shear rate and wall shear stresses, based on the finite element method. Dependence of the flow on the severity of stenosis is investigated.

\section{Mathematical model}

Precise blood flow analysis requires simulating the flow of blood through the lumen and the various layers in deforming blood vessels. However, to capture the main feature of blood flow and to keep the model simple, in this work the flow through the intimal and media layers and the deformation of blood vessels are neglected.

Generally, when the shear rate is greater than $100 \mathrm{~s}^{-1}$, blood behaves as an incompressible Newtonian fluid $[5,6,7]$. However, when the shear rate is lower than $100 \mathrm{~s}^{-1}$, blood behaves as a non-Newtonian fluid and the stresses depend on the deformation rate nonlinearly. Various non-Newtonian models 
have been proposed including the power law model, the Carreau model. In all these models, the stresses are related to the deformation rate by

$$
\sigma_{i j}=-p \delta_{i j}+\mu_{n}\left(u_{i, j}+u_{j, i}\right),
$$

which is similar to the Newtonian model except that the viscosity is the function of shear rate instead of a constant. In different non-Newtonian models, there are different relations between the viscosity $\mu_{n}$, and the shear rate

$$
\dot{\gamma}=\sqrt{2 \operatorname{tr}\left[\frac{1}{2}\left(u_{i, j}+u_{j, i}\right)\right]^{2}} .
$$

This article focuses on blood flow in the artery lumen. We assume that blood is an incompressible non-Newtonian fluid. The Carreau model is used to determine the viscosity of blood, $\mu_{n}=\mu_{\infty}+\left(\mu_{0}-\mu_{\infty}\right)\left[1+(\lambda \dot{\gamma})^{2}\right]^{(n-1) / 2}$, where $\mu_{0}$ is the zero shear viscosity, $\mu_{\infty}$ is the infinite shear viscosity and $n$ is a parameter between 0 and 1 . In this study $\mu_{0}=0.56 \mathrm{~g} \mathrm{~cm}^{-1} \mathrm{~s}^{-1}, \mu_{\infty}=$ $0.0345 \mathrm{~g} \mathrm{~cm}^{-1} \mathrm{~s}^{-1}, \lambda=3.313 \mathrm{~s}$ and $n=0.3568$. The equations governing the blood flow include the constitutive equation (1) and the following continuity equation and the stress equations of motion,

$$
\begin{aligned}
& u_{i, i}=0, \\
& \rho\left(\frac{\partial u_{i}}{\partial t}+u_{j} u_{i, j}\right)=\frac{\partial \sigma_{j i}}{\partial x_{j}}+F_{i},
\end{aligned}
$$

where we use the index notation with repeated literal index representing summation over the index range, $\rho$ denotes the blood density which is $1.06 \mathrm{~g} \mathrm{~cm}^{-3}$, $u_{i}$ represents the component of velocity vector in the $i$ th direction, $p$ denotes pressure in the channel, and $F$ is the body force acting on the fluid.

To specify the boundary conditions for the problem, we consider precisely the blood flow mechanism. The heart is a two step pump: first the atria, then the ventricles contract. The heart ejects and fills with blood in alternating cycles known as systole and diastole. Blood is ejected from the left ventricle 
TABLE 1: Values of parameters used in computational region.

\begin{tabular}{rrrrr}
\hline$n$ & $\alpha_{n}^{Q}$ & $\theta_{n}^{Q}$ & $\alpha_{n}^{p}$ & $\theta_{n}^{p}$ \\
\hline 1 & 17.28 & 2.256 & -21.740 & -0.406 \\
2 & -34.91 & -0.226 & -9.088 & 0.202 \\
3 & -16.11 & 1.228 & 4.771 & -0.633 \\
4 & 11.70 & 4.882 & 2.035 & -4.315 \\
5 & 6.64 & -0.074 & 0.768 & 3.932 \\
\hline
\end{tabular}

into the arterial system during systole. The heart rests during diastole in which no blood is ejected. The cyclic nature of the heart pump creates pulsatile conditions in all the arteries.

Ignoring the variation of cardiac period, the flow rate and the pulsatile pressure are determined by the following Fourier series representations

$$
\begin{aligned}
& Q(t)=\bar{Q}+\sum_{n=1}^{5} \alpha_{n}^{Q} \cos \left(\frac{2 n \pi t}{T}-\theta_{n}^{Q}\right), \\
& p_{0}(t)=\bar{p}+\sum_{n=1}^{5} \alpha_{n}^{p} \cos \left(\frac{2 n \pi t}{T}-\theta_{n}^{p}\right),
\end{aligned}
$$

where $\bar{Q}=59.09 \mathrm{~cm}^{3} / \min$ and $\bar{p}=122.5 \mathrm{mmHg}$ are respectively the mean flow rate and mean pressure, $T=0.75 \mathrm{~s}$ is the cardiac period, and the values of $\alpha_{n}^{Q}, \alpha_{n}^{p}, \theta_{n}^{Q}$ and $\theta_{n}^{p}$ are listed in Table 1 [8].

We therefore impose a pulsatile flow rate condition on the inlet boundary $\partial \Omega_{\text {in }}$ and a corresponding pulsatile pressure condition on the outlet boundary $\partial \Omega_{\text {out }}$ of the computational region.

Now by substituting equations (1) into (3), we obtain the following Navier- 
Stokes equations

$$
\rho\left(\frac{\partial u_{i}}{\partial t}+u_{j} u_{i, j}\right)=F_{i}-p_{, i}+\left[\mu_{n}\left(u_{i, j}+u_{j, i}\right)\right]_{, j}
$$

which together with the continuity equation (2) constitute a closed system of four partial differential equations in terms of four coordinate and time dependent unknown functions $u_{1}, u_{2}, u_{3}$ and $p$. The system, supplemented by the initial condition and boundary conditions, is solved numerically to yield the velocity field and the pressure distribution and consequently the shear stresses on the arterial wall.

\section{Method of solution}

To develop the variational statement for the boundary value problem, we consider the following integral representation of the problem.

Find $u_{1}, u_{2}, u_{3}$ and $p \in H_{\Omega}^{1}$ such that for all test functions $\hat{u}_{1}, \hat{u}_{2}, \hat{u}_{3} \in$ $H_{0 \mathbf{u}}^{1}(\Omega)$ and $\hat{p} \in H^{1}(\Omega)$, all the Dirichlet boundary conditions for the unknown functions are satisfied and

$$
\begin{aligned}
& \left(u_{i, i}, \hat{p}\right)=0, \\
& \left(\rho \frac{\partial u_{i}}{\partial t}, \hat{u}_{i}\right)+\left(\rho u_{j} u_{i, j}, \hat{u}_{i}\right)-\left(\left[\mu_{n}\left(u_{i, j}+u_{j, i}\right)\right]_{, j}, \hat{u}_{i}\right)+\left(p_{, i}, \hat{u}_{i}\right)=\left(F_{i}, \hat{u}_{i}\right),
\end{aligned}
$$

where $(\cdot, \cdot)$ denotes the inner product on the square integrable function space $L^{2}(\Omega), H^{1}(\Omega)$ is the Sobolev space $W^{1,2}(\Omega)$ with norm $\|\cdot\|_{1,2, \Omega}, H_{0 u}^{1}(\Omega)=$ $\left\{v \in H^{1}(\Omega) \mid v=0\right.$ on $\partial \Omega_{\text {in }}$ and $\left.\partial \Omega_{\text {wall }}\right\}$. A standard procedure is then carried out to reduce the second order derivatives involved in the above problem into the first order ones using integration by parts to ensure that all integrals involved are well defined. 
To find the numerical solution of the problem, we pose the variational problem into an $N$-dimension subspace. The computation domain $\Omega$ is discretized into a finite number of elements connected by $N$ nodes. Let $\mathbf{U}$ and $\mathbf{P}$ denote respectively the global vectors with each $i$ th entry representing the value of the corresponding unknown function at the $i$ th node of the finite element mesh. Then, by using the Galerkin finite element formulation, we obtain the following systems of ordinary differential equations:

$$
\begin{aligned}
& D_{u} \mathbf{U}=0, \\
& M \dot{\mathbf{U}}+A_{u} \mathbf{U}+A_{p} \mathbf{P}=\mathbf{F},
\end{aligned}
$$

where the superposed dot represents differentiation with respect to time and all coefficient matrices are global matrices assembled from the element matrices. Matrix $M$ corresponds to the transient term, matrices $A_{u}$ and $D_{u}$ correspond to the advection and diffusion terms, matrix $A_{p}$ corresponds to the pressure term and vector $\mathbf{F}$ provides the forcing functions for the NavierStoke equations. A standard backward Euler scheme is then used to solve the above system of ordinary differential equations to determine the velocity and pressure fields at any instant of time.

\section{Numerical results and discussion}

The examples under consideration are stenotic arteries with 50\%-, 65\%- and $75 \%$-area severity. The 3D geometries are straight tubes of length of $5 \mathrm{~cm}$ and diameter of $0.210 \mathrm{~cm}$ in which a stenosis with spherical curvature is present in the middle part on one side of the internal wall as shown in Figure 1.

The computational domain $\Omega$ is bounded by the boundary $\partial \Omega=\partial \Omega_{\text {in }} \cup$ $\partial \Omega_{\text {wall }} \cup \partial \Omega_{\text {out }}$. As numerical error decreases as element size decreases, a grid resolution study is carried out to investigate the effect of finite element mesh on numerical results. The results show that the solution converges when the number of element reaches 11,836 elements. Hence, in this study, 


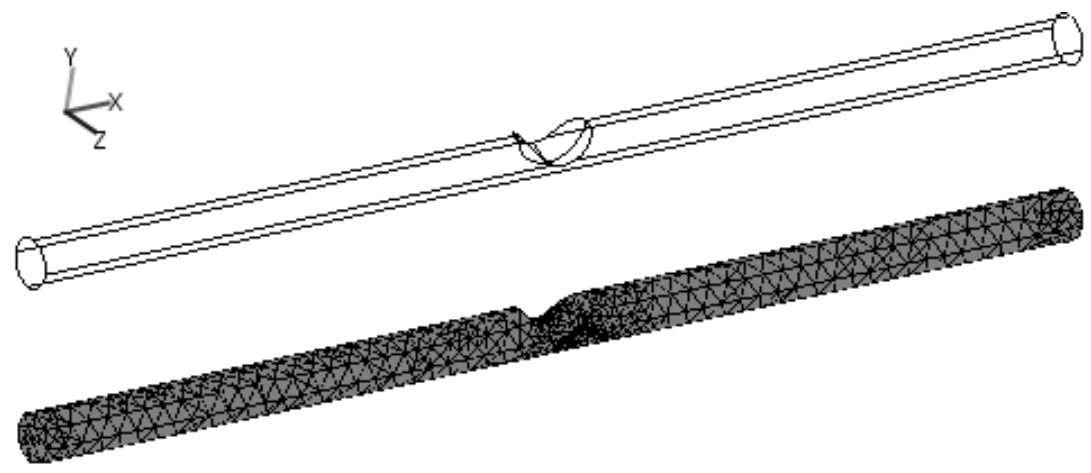

Figure 1: The finite element mesh of the $75 \%$ stenotic tubes.

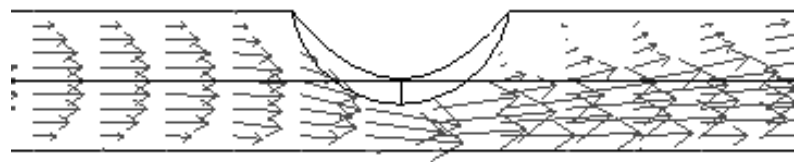

(a) Vector plot: Velocity profiles $(\mathrm{cm} / \mathrm{s})$.

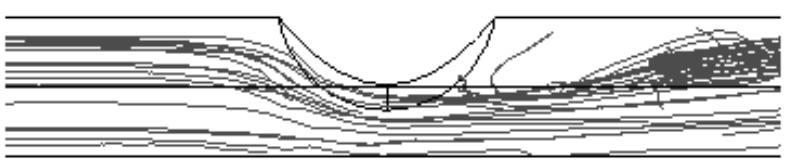

(b) Streamline: Velocity field.

FiguRE 2: Velocity profiles and streamlines in the Oxy plane for the $75 \%$ stenotic tube at the peak of systole. 
the 3D geometry tubes are discretized into 11,836 quadratic tetrahedron elements with 68,858 degrees of freedom (velocity and pressure). The solutions were computed for five cardiac cycles to ensure reproducibility of the pulsed characteristic flow. The time step size $\Delta t$ used for each model is allowed to have different values. For the case with more percentage area severity, we use smaller time step size. For the case of 50\%-area severity, the minimum time step is taken to be $\Delta t_{\min }=0.005 \mathrm{~s}$, the maximum time step is $\Delta t_{\max }=0.01 \mathrm{~s}$, and for $65 \%$ - and $75 \%$-area severity, the time steps are changed to $\Delta t_{\min }=0.001 \mathrm{~s}, \Delta t_{\max }=0.005 \mathrm{~s}$.

To determine the inlet pulsatile flow rate and outlet pulse pressure, the parameters listed in Table 1 are used. Figure 2 depicts the longitudinal velocity vectors and streamlines at the peak of the systole in the Oxy plane for the $75 \%$ stenotic tube. The plot clearly shows the flow pattern. Upstream from the stenosis, the velocity profile in the $x$-direction is parabolic as shown in Figure 3, and the fluid passes through the stenosis at high speed, especially at the throat of the stenosis. Downstream from the stenosis region, the distal part, the flow has stair-step shape profile and the longitudinal velocity $u_{x}$ is negative (along the negative $x$ direction) in the recirculation region. A region of reversal flow occurs at the downstream, next to the stenosis whereas the jet impinging occurs at the throat of the stenosis. Higher area blockage severity leads to larger pressure dropping around the stenosis and consequently gives higher speed in the stenosis area.

The relation between the blood pressure and blood velocity field are demonstrated in Figure 4. These figures illustrate the pressure distribution along a longitudinal line and the mean flow velocity of blood at the throat line during the systolic periods. It shows that the pressure drops very quickly near the stenosis site and creates a jet flow at the throat of the stenosis. The results also show that a slightly increasing zone of ascending motion is present in the upstream zone. When the flow reaches the stenosis site, the pressure suddenly drops causing a suddenly increasing blood velocity of $519.931 \mathrm{~cm} / \mathrm{s}$ at the peak of the systole and $450.171 \mathrm{~cm} / \mathrm{s}$ at the peak of 


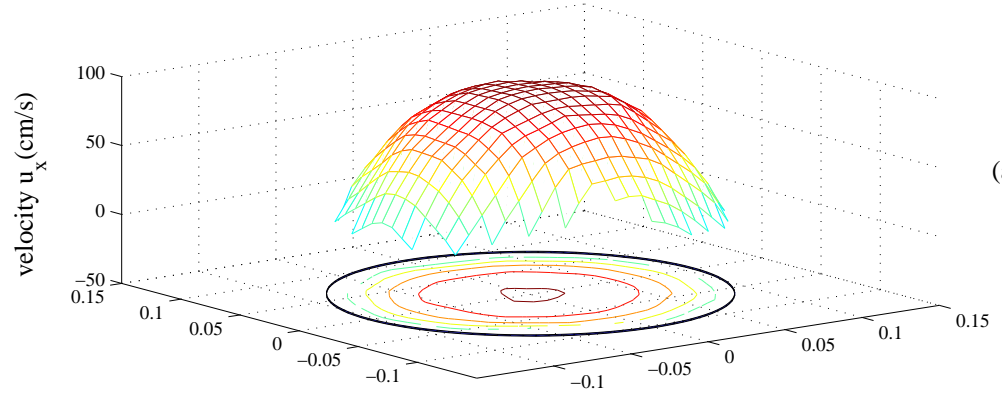

(a)

radial axis

radial axis

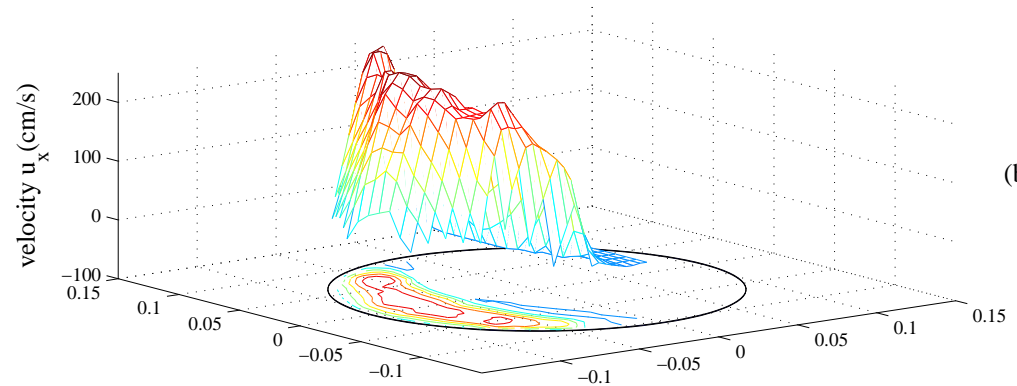

radial axis

radial axis

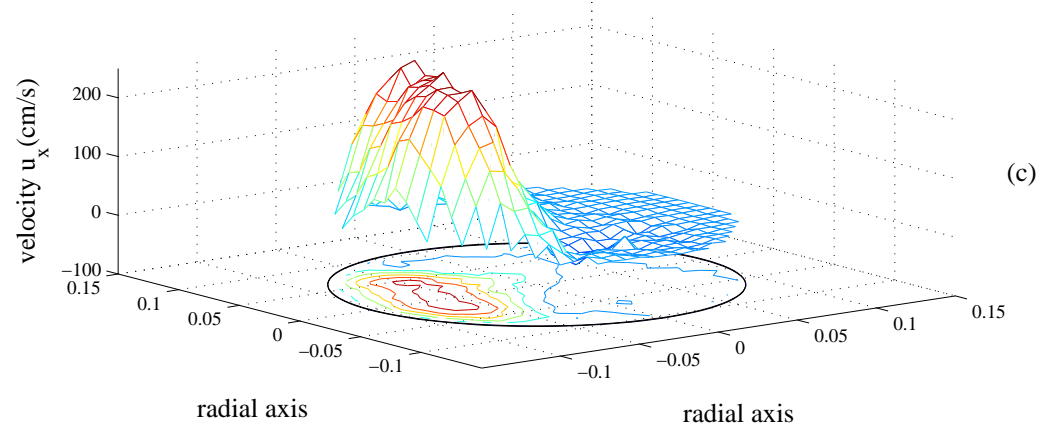

FigurE 3: The velocity $u_{x}$ at the peak of systole in the $O y z$ plane of the $75 \%$ stenotic tube: (a) at the upstream cross section $x=1.5 \mathrm{~cm}$, (b) at the throat cross section $x=2.5 \mathrm{~cm}$, and (c) at the downstream cross section $x=2.7 \mathrm{~cm}$ 

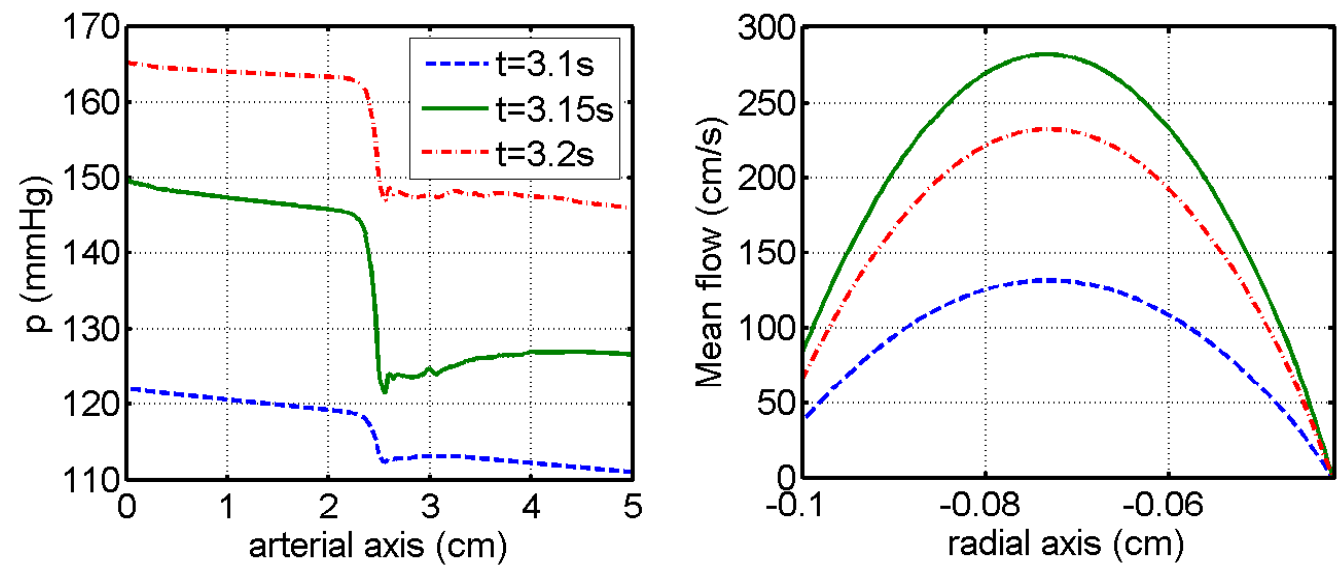

Figure 4: Pressure along a longitudinal line and mean flow at the throat line of $75 \%$ stenotic tube during the systolic period.

the diastole. Near the stenosis site, the flow accelerates ( $d Q / d t$ is positive) when a negative pressure gradient exists. In the downstream zone, the flow decelerates and an adverse pressure gradient exists. This means that an extra higher pressure jump is created to impel the flow passing through a narrowing channel. Figure 5 shows the time characteristics of the pulsatile flow in terms of mean flow, pressure and shear rate. In a healthy artery, the wall shear stress is approximately $15 \mathrm{dyn} / \mathrm{cm}^{2}$ [9]. To determine the critical flow condition, measurement of wall shear stress using numerical experiments becomes necessary. The shear rate and wall shear stress were computed for a model with 75\%-area severity. The magnitude of shear rate along a longitudinal line increases sharply before the occlusion is approached and reaches a maximum value near the center of the throat around $2 \times 10^{4} \mathrm{~s}^{-1}$ at the peak of the systole and at $8 \times 10^{3} \mathrm{~s}^{-1}$ at the peak of the diastole. It then decreases in the downstream. To depict the wall shear stress along the arterial wall, we plot the solution on the plane representing the wall surface where the stenosis is located at the center. The direction of the wall shear stress oscillates in the recirculation zone at downstream as shown in Figure 6. 

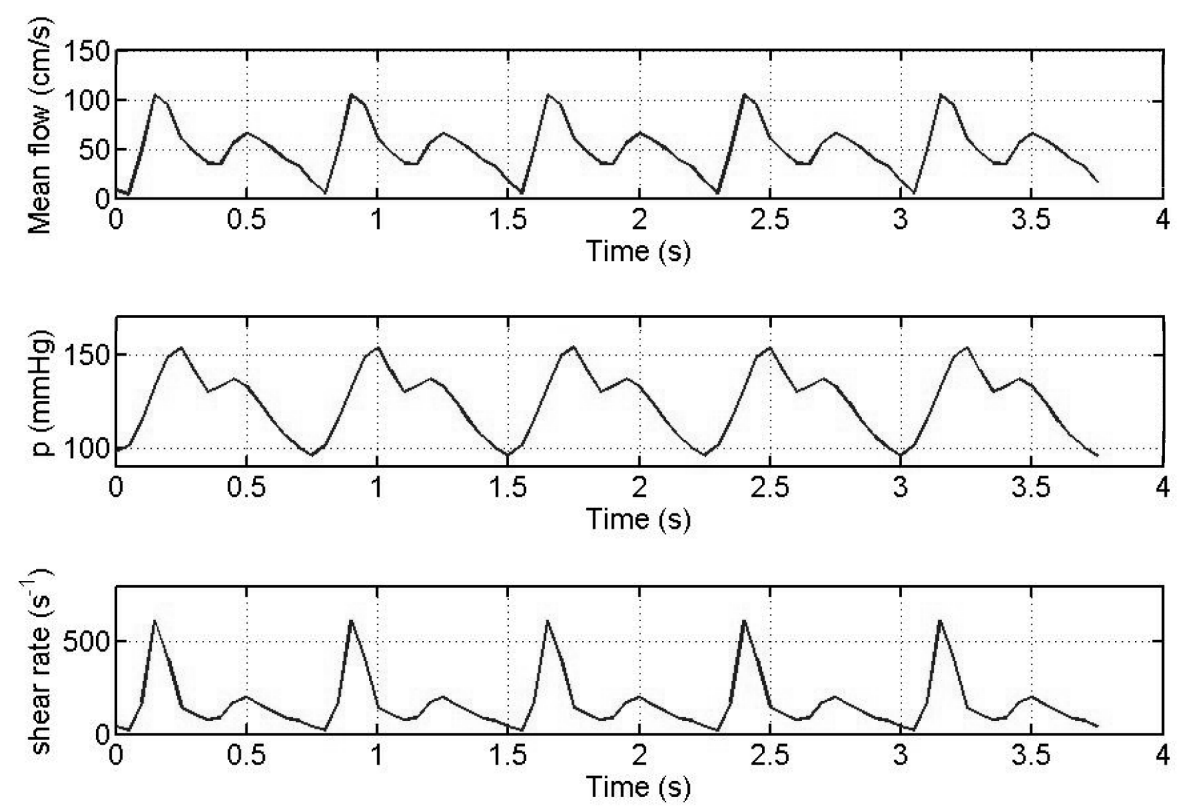

Figure 5: Pulsatile flow velocity, pulse pressure, and variation of shear rate with respect to time at an upstream point $(2.3,0,0)$ for a $50 \%$ stenotic tube. 

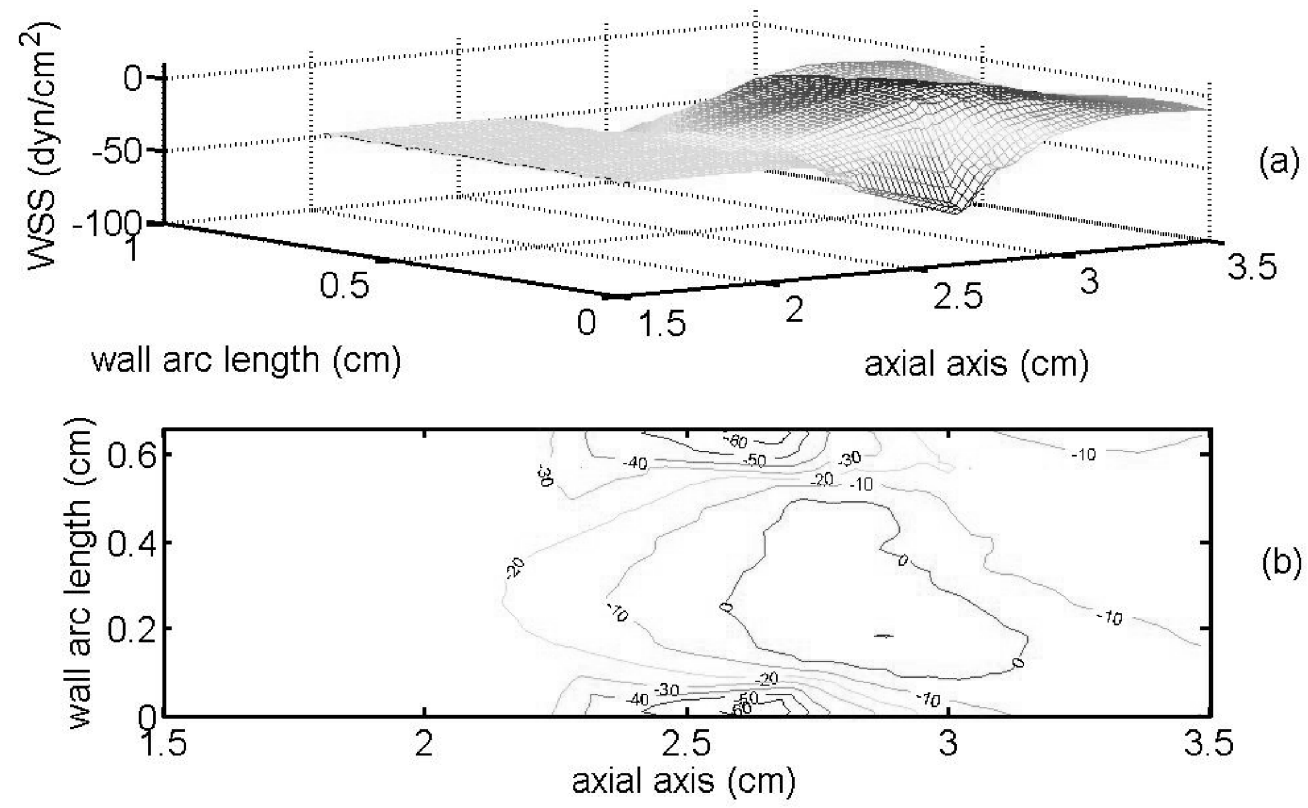

(b)

Figure 6: Wall shear stress at the end of diastole $t=3.70 \mathrm{~s}$ : (a) surface plot, (b) contour plot.

Comparing the results obtained from three stenotic tubes with 50\%-, 65\%and $75 \%$-area severity, we find that higher percent-area severity of stenosis leads to higher extra pressure jumps, higher blood speeds around the stenosis site, higher shear rate and higher wall shear stress.

\section{Conclusions}

A mathematical model for simulating the blood flow in stenotic arteries has been constructed. The model is used to study the critical flow in stenotic 
arteries with three different severities of $50 \%, 65 \%$ and $75 \%$. A rapid fall in pressure is observed as the occlusion is approached. Higher percentage area severity leads to greater pressure drops around the stenosis. It also leads to higher speeds in the stenosis area. The results clearly show the relation between pressure and velocity field. The flow is accelerating when a negative pressure gradient exists at the stenosis site. The flow decelerates when an adverse pressure gradient exists. This extra pressure jump helps to impel the flow passing through the narrowing channel. The results also show a similar pattern in the pulsatile velocity, in the pulse pressure and in the variation of shear rate in cardiac cycles. These confirm the features of the characteristic of the periodic motion. Therefore, in the presence of a narrowing vessel lumen with different area severity, the flow experiences resistance, which causes an increase in the shear stress and in the pressure drop. Higher percent-area severity of stenosis produces a higher pressure drop, a higher blood speed, a higher shear rate and a higher wall shear stress.

Blood flow in a small stenotic artery is an extremely complex phenomenon. There are many unresolved modeling problems such as the flow in the arterial wall which is deformed during the cardiac period. The presented work only focuses on blood flow in the lumen channel which does not include the effect of the wall. Further work will be carried out to incorporate fluid-wall interaction in the stenotic artery.

Acknowledgements The project is supported by the 2005 Endeavour Australia Chueng Kong Awards and the Mahidol University Research funds.

\section{References}

[1] D. N. Ku, Blood flow in arteries, Annual Review of FLuid Mechanics 29 (1997) 399-434. C1007 
[2] D. Mann and J. Tarbell, Flow of non-Newtonian blood analog fluids in rigid curved and straight artery models, Biorheology 27 (1990) 711-733. C1008

[3] M. Grigioni, C. Daniele and G. D'Avenio, The role of wall shear stress in unsteady vascular dynamics, Progress in Biomedical Research 7 (3) (2002) 204-212. C1008

[4] K. B. Chandran, J. H. Mun, K. K. Choi, J. S. Chen, A. Hamilton, A. Nagaraj and D. D. McPherson, A method for in-vivo analysis for regional arterial wall material property alterations with alterosclerosis: preliminary results, Medical Engineering and Physics 25 (2003) 289-298. C1008

[5] M. Bonert, J. G. Myers, S. Fremes, J. Williams and C. R. Ethier, A numerical study of blood flow in coronary artery bypass graft Side-to-Side Anastomoses, in Annals of Biomedical Engineering 30 (2002) 599-611. C1008

[6] D. Y. Fei, J. D. Thomas and S. E. Rittgers, The effect of angle and flow rate upon hemodynamics in distal vascular graft anastomoses: a numerical model study, Journal of Biomechanical Engineering 116 (1994) 331-336. C1008

[7] M. H. Song, M. Sato, Y. Ueda, Three dimensional simulation of coronary artery bypass grafting with the use of computational fluid dynamics, Surg Today 30(2000), 993-998. C1008

[8] B. Wiwatanapataphee, D. Poltem, Y. H. Wu and Y. Lenbury, Simulation of pulsatile flow of blood in stenosed coronary artery bypass with graft, Mathematical Biosciences 3 (2) (2006) 371-383. C1010

[9] S. Glagov and C. K. Zarins and D. P. Giddens and D. N. Ku, Hemodynamics and Atherosclerosis, Insights and perspectives gained from studies of human arteries, Archieves of Pathology and Laboratory Medicine 112 (1988) 1018-1031. C1016 


\section{Author addresses}

1. B. Wiwatanapataphee, Department of Mathematics, Mahidol University, Bangkok 10400, ThaILAND. mailto:scbww@mahidol.ac.th

2. Y.-H. Wu, Department of Mathematics and Statistics, Curtin University of Technology, Perth, Western Australia 6845, Australia. mailto:yhwu@maths . curtin.edu . au

3. S. Amornsamankul, Department of Mathematics and Statistics, Curtin University of Technology, Perth, Western Australia 6845, Australia.

4. B. Novaprateep, Department of Mathematics, Mahidol University, Bangkok 10400, ThaILAND. 